incident HIV cases nationwide (2008). Although Trichomonas vaginalis (TV) infection has been associated with an increased risk of HIV acquisition in women, the prevalence of TV in Miami is unknown due to lack of routine screening and low sensitivity testing methods. The purpose of this study is to establish a prevalence of TV in women seeking services at the Miami-Dade County Health Department Downtown STD Clinic compared to the prevalence of routinely screened STDs, gonorrhoea (GC) and Chlamydia (CT).

Methods This study analyses baseline data from the Miami site of Project AWARE which is a multi-centre randomised clinical trial that seeks to test the effectiveness of risk reduction counselling in preventing sexually transmitted infections including HIV among HIV-negative persons. Eligibility criteria for Project Aware included negative or unknown HIV status, age of 18 , and ability to provide informed consent. We recruited 251 women aged 18-64 for STD screening. The screen included FDA-approved Aptima GC/CT NAAT (nucleic acid amplification test) and a newly validated Aptima TV NAAT. Specimens were collected by vaginal swab performed either by a clinician or the patient. Additionally, we report the TV diagnoses given the current clinic protocol in which only symptomatic women received a wet mount analysed by light microscopy. We use descriptive statistics to report the prevalence of GC, CT and TV (as diagnosed by NAAT and wet mount) in our clinic.

Results Of the 251 women in our study, 163 high-risk populations. Received a wet mount. By wet mount, we found a $9 \%$ prevalence of TV in our patients. Comparatively, when all 251 patients were screened using NAAT, the TV prevalence was $20 \%$. The prevalence of CT was $14 \%$ and GC was $6 \%$.

Conclusions TV is the most prevalent STD in patients in our clinic which draws upon high-risk individuals in urban Miami. TV infections were greater than CT, often believed to be the most prevalent STD in our population. Further, the routine screening using the TV NAAT detected 27 cases of TV that would have been undiagnosed given current clinic protocol. Since Miami, FL has the highest prevalence of HIV in the country and TV infection has been linked to new HIV infection, we believe that routine TV screening by NAAT should be instituted in high-risk populations.

\section{P1-S1.12 AGE-SPECIFIC INCIDENCE OF CHLAMYDIA, GONORRHOEA, AND TRICHOMONIASIS INFECTION IN ADOLESCENT WOMEN}

doi:10.1136/sextrans-2011-050108.12

${ }^{1} \mathrm{~W}$ Tu, ${ }^{1} \mathrm{~B}$ Batteiger, ${ }^{1} \mathrm{~S}$ Ofner, ${ }^{2} \mathrm{~B}$ Van Der Pol, ${ }^{1} \mathrm{D}$ Fortenberry. ${ }^{1}$ Indiana University, School of Medicine, Indianapolis, Indiana, USA; ${ }^{2}$ Indiana University, Indiana, USA

Background Age-specific incidence rates of sexually transmitted infections (STI) with Chlamydia trachomatis (CT), Neisseria gonorrhoeae (GC), and Trichomonas vaginalis (TV) are not well characterised in adolescents and young adult women. In this research, we described the CT, GC, and TV incidence rates in young women as functions of age.

Methods Young women aged 14 and 17 were recruited from three adolescent medicine clinics in Indianapolis, Indiana. Study participants were followed longitudinally for up to 8.2 years. Participants were tested for CT, GC, and TV at the time of enrolment, and at subsequent quarterly visits. Infected individuals were treated at or shortly after each visit. We analysed the longitudinally measured incidence infections with CT, GC, and TV using generalised additive mixed effect models (GAMM) with a logit link for binary outcomes. The age effect on STI incidence was modelled as a smooth nonlinear function in the GAMM analysis and a random subject effect was included to accommodate the correlations among repeated STI assessments within each individual. Estimated incidence rates (with $95 \%$ CIs, in colour) for each organism were reported as smooth functions of age.

Results The cohort included 386 young women. The average age at enrolment of study participants was 15.3 years ( $S D=1.1$ years). A majority (89.1\%) of study participants were African-American. The average age at first sexual intercourse was 14.2 years $(\mathrm{SD}=2$, median $=14$ years). The mean cumulative number of sexual partners measured at the time enrolment was $3(\mathrm{SD}=4$, median=2). The average length of follow-up was 3.5 years ( $\mathrm{SD}=2.2$ years). Baseline prevalence rates were $10.9 \%, 4.4 \%$ and $6.0 \%$ for CT, GC, and TV, respectively. The age-specific incidence rates for the three organisms and any STI are seen in the Abstract P1-S1.12 figure 1.
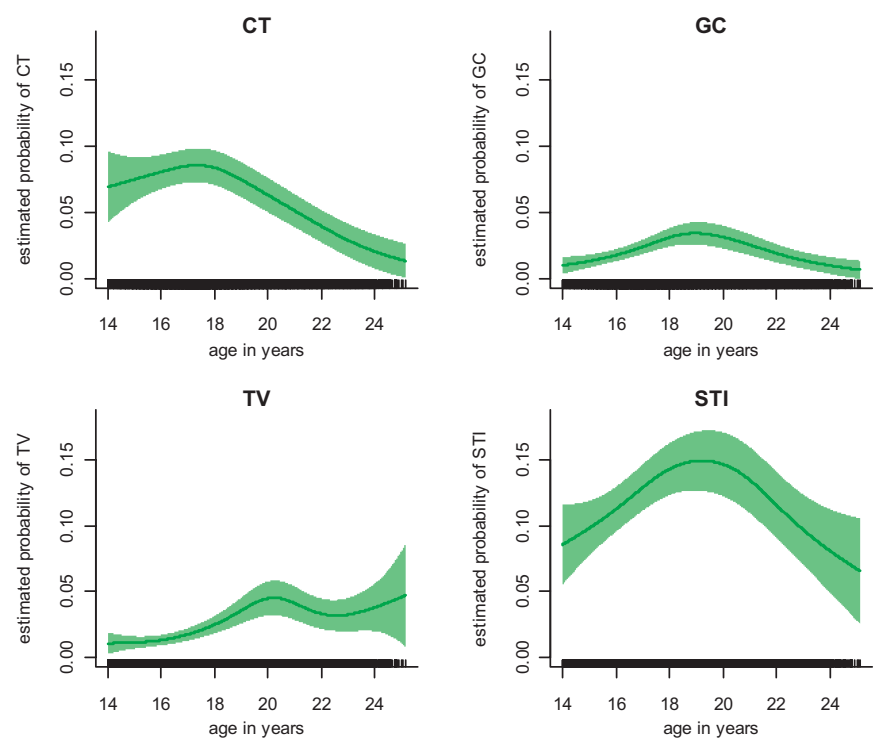

Abstract P1-S1.12 Figure 1 WTU.

Conclusions The estimated STI incidence rates clearly differ by organisms, not only in magnitude but also in peak age. While the prevalence rates of the respective organisms in the partner population may be a contributor to the differential risk of STI acquisition, the fact that such differences were observed from the same group of individuals with the same sexual partners and sexual behaviours raises questions about age-related differences in susceptibility to infection by the three organisms.

\section{Epidemiology poster session 1: STI trends-HIV}

\section{P1-S1.13 HETEROGENEITY OF THE HIV EPIDEMIC IN THE GENERAL POPULATION OF KARNATAKA STATE, INDIA}

doi:10.1136/sextrans-2011-050108.13

${ }^{1} \mathrm{P}$ Banandur, ${ }^{2} \mathrm{R}$ S Potty, ${ }^{3} \mathrm{~S}$ B Mahagaonkar, ${ }^{4} \mathrm{~J}$ Bradley, ${ }^{5} \mathrm{R}$ M Banadakoppa, ${ }^{6} \mathrm{R}$ G Washington, ${ }^{7} \mathrm{~J}$ F Blanchard, ${ }^{7} \mathrm{~S}$ Moses, ${ }^{8} \mathrm{C} \mathrm{M}$ Lowndes, ${ }^{9} \mathrm{M}$ Alary. ${ }^{7} \mathrm{CHARME}$ ॥ Project, Rajarajeswari Medical College and Hospital, Bangalore, India; ${ }^{2}$ CHARME II Project, Bangalore, India; ${ }^{3} \mathrm{CHARME}$ I Project, India, Karuna Medical College, India; ${ }^{4}$ CHARME I Project, Bangalore, India; ${ }^{5}$ Karnataka Health Promotion Trust, Bangalore, India; ${ }^{6}$ St Johns Research Institute, India; ${ }^{7}$ University of Manitoba, Winnipeg, Canada; ${ }^{8}$ Health Protection Agency, UK; ${ }^{9}$ Centre hospitalier affilié universitaire de Québec, Quebec, Canada

Background In the context of AVAHAN-The India AIDS Initiative of the Bill and Melinda Gates Foundation, general population surveys (GPS) have been carried out in three Karnataka districts in India. Given the north-south gradient already observed in HIV 
prevalence in antenatal population in Karnataka districts, we analysed data from three such surveys to detect heterogeneity. The three districts analysed here are Belgaum (Northern Karnataka), Bellary (middle of the state) and Mysore (Southern Karnataka).

Methods We conducted a comparative analysis of these three GPS conducted between 2005 and 2007. Subjects were selected using a two-stage cluster sampling design with equal number of rural and urban participants, and of men and women, with a target sample size of 6000 per district. Questionnaires on socio-demographic factors and HIV risk behaviour were administered. Blood samples were tested for HIV, syphilis and HSV-2 antibodies, whereas urine samples were tested for gonorrhoea and chlamydia using nucleic acid amplification tests. A descriptive analysis of prevalence of HIV and sexually transmitted infections (STIs) was conducted according to age, district and place of residence (rural/urban).

Results Abstract P1-S1.13 table 1 shows HIV prevalence per district according to gender and place of residence. Belgaum had the highest overall HIV and HSV-2 prevalence (16.9\%) and the lowest prevalence of curable STIs (Chlamydia- $0.38 \%$; Syphilis- $0.42 \%$ ) among the three districts. Women in Belgaum had a higher HIV prevalence $(\mathrm{OR}=2.16,95 \% \mathrm{CI}-1.02$ to 4.58$)$ compared to women in Mysore. The HIV epidemic in Belgaum is predominantly rural and among women. In Bellary, it is predominantly urban and among men. Mysore had the lowest prevalence of HIV and HSV-2 (10.9\%) and the highest prevalence of curable STIs (Chlamydia-1.05\%; Syphilis-1.38\%) among the three districts. There were only six cases of gonorhoea in total (five in Mysore and one in Bellary).

Abstract P1-S1.13 Table 1 Gender specific prevalence of HIV by district and place of residence

\begin{tabular}{llll}
\hline Place of residence & Belgaum \% $(95 \% \mathbf{C I})$ & Bellary \% $(\mathbf{9 5 \%} \mathrm{Cl})$ & Mysore \% $(95 \% \mathrm{CI})$ \\
\hline All total & $1.43(0.86$ to 2.01$)$ & $1.18(0.74$ to 1.62$)$ & $0.80(0.50$ to 1.09$)$ \\
Males & $1.28(0.51$ to 2.06$)$ & $1.24(0.70$ to 1.78$)$ & $0.98(0.45$ to 1.52$)$ \\
Females & $1.58(0.94$ to 2.23$)$ & $1.13(0.59$ to 1.66$)$ & $0.65(0.28$ to 1.02$)$ \\
& & & \\
Urban total & $0.63(0.18$ to 1.09$)$ & $1.36(0.65$ to 2.07$)$ & $0.94(0.49$ to 1.39$)$ \\
Urban males & $0.69(0.25$ to 1.12$)$ & $1.64(0.71$ to 2.56$)$ & $1.06(0.35$ to 1.78$)$ \\
Urban females & $0.58(0.00$ to 1.16$)$ & $1.12(0.37$ to 1.86$)$ & $0.84(0.34$ to 1.35$)$ \\
& & & \\
Rural total & $1.69(0.90$ to 2.49$)$ & $1.05(0.46$ to 1.64$)$ & $0.71(0.31$ to 1.10$)$ \\
Rural males & $1.48(0.41$ to 2.55$)$ & $0.97(0.29$ to 1.65$)$ & $0.93(0.13$ to 1.74$)$ \\
Rural females & $1.90(1.01$ to 2.79$)$ & $1.13(0.34$ to 1.93$)$ & $0.51(0.00$ to 1.05$)$ \\
\hline
\end{tabular}

${ }^{*}$ All figures in weighted percentages, $95 \% \mathrm{Cl}$.

Conclusion The HIV epidemic in Karnataka shows considerable heterogeneity. This analysis validates the observed north-south gradient. The sex work structure in these three districts might explain the heterogeneity of the HIV epidemic in these three districts. Higher prevalence of HIV and HSV-2 and lower prevalence of curable STIs in Belgaum suggests a late epidemic phase. Mysore may have an early phase epidemic with higher prevalence of curable STIs.

\section{P1-S1.14 IS HIV PREVALENCE DECLINING IN SOUTHERN INDIA? EVIDENCE FROM TWO ROUNDS OF GENERAL POPULATION SURVEYS IN BAGALKOT DISTRICT, KARNATAKA}

\section{doi:10.1136/sextrans-2011-050108.14}

\begin{abstract}
${ }^{1} \mathrm{~S}$ P Rajaram, ${ }^{2} \mathrm{~J}$ E Bradley, 'B M Ramesh, ' $\mathrm{S}$ Isac, ${ }^{1} \mathrm{R}$ G Washington, ${ }^{3} \mathrm{~S}$ Moses, ${ }^{3} \mathrm{~J}$ F Blanchard, ${ }^{3} \mathrm{M}$ Becker, ${ }^{2} \mathrm{M}$ Alary. ${ }^{1}$ Karnataka Health Promotion Trust, Bangalore, India; ${ }^{2}$ Centre hospitalier affilié universitaire de Québec, Quebec, Canada; ${ }^{3}$ University of Manitoba, Winnipeg, Canada
\end{abstract}

Background As a part of the evaluation of Avahan, the India AIDS initiative of the Bill \& Melinda Gates Foundation, a cross-sectional survey was undertaken in the general population of Bagalkot district, Karnataka state, South India, in 2009. This replicated an earlier survey in 2003 that had examined HIV prevalence and risk behaviours.

Methods The repeat survey in 2009 was conducted in the same rural and urban areas as the 2003 study. The study covered 10 rural villages and 20 urban blocks of three of the six talukas (sub-district units) in the district. In both surveys, a target sample of 6600 adult males and females was selected. Urine and blood samples were collected from all consenting participants for HIV and STI testing. An individual was deemed HIV positive, if positive on two different tests. We compared HIV and STI prevalence in 2003 and 2009. We also examined the age-specific distribution of HIV prevalence among rural and urban males and females at both time points. These analyses used logistic regression that considers survey design to adjust for characteristics of the population that may have changed between the two rounds.

Results Overall, HIV prevalence in the district declined from $3.16 \%$ in 2003 to $2.58 \%$ in 2009, although this decline was not statistically significant $(\mathrm{OR}=0.82, \mathrm{p}=0.278)$. The prevalence of active syphilis was $<1 \%$ and was similar in both periods. A slight decline in the prevalence of HSV-2 was observed over the period, but the difference was not statistically significant. There was a significant decline observed in HIV prevalence among persons aged 15-24, among persons below 30 years in urban areas, and among all women younger than 20 years of age (Abstract P1-S1.14 table 1). Among rural males aged 40 and above, we observed a significant increase in HIV prevalence from 0.71 to 5.33 (Abstract P1-S1.14 table 1), a level similar to that found in the younger age group in 2003.

Abstract P1-S1.14 Table 1 HIV prevalence and adjusted OR among selected population groups

\begin{tabular}{lllll}
\hline Sub-group & $\begin{array}{l}\text { Round 1, } \\
\text { 2003 (\%) }\end{array}$ & $\begin{array}{l}\text { Round 2, } \\
\mathbf{2 0 0 9}(\%)\end{array}$ & $\begin{array}{l}\text { Adjusted 0R, 2009 vs } \\
\mathbf{2 0 0 3 ~ ( 9 5 \% ~ C l ) ~}\end{array}$ & p Value \\
\hline Overall & 3.16 & 2.58 & $0.82(0.57$ to 1.18$)$ & 0.278 \\
Age 15-24 & 2.4 & 1.26 & $0.54(0.30$ to 0.96$)$ & 0.038 \\
Age 15-29, urban & 2.04 & 0.77 & $0.36(0.20$ to 0.63$)$ & 0.001 \\
Age 15-19, female & 2.25 & 0.49 & $0.15(0.04$ to 0.61$)$ & 0.009 \\
Age 40+, rural males & 0.71 & 5.33 & $13.80(2.59$ to 73.45$)$ & 0.006 \\
\hline
\end{tabular}

Conclusion We observed a downward overall trend in HIV prevalence in Bagalkot district over the period examined, and a significant decline in HIV prevalence among younger age groups, where HIV prevalence more closely reflects incidence. The increase in HIV prevalence among older rural males may have been due to a cohort effect, as they aged over time. This may also have in part reflected increased survival in those age groups, as a result of the scaling-up of antiretroviral treatment programs throughout the state.

\section{P1-S1.15 THE STATUS OF THE HIV EPIDEMIC IN LEBANON-SYSTEMATIC REVIEW AND SYNTHESIS}

doi:10.1136/sextrans-2011-050108.15

${ }^{1} \mathrm{G}$ Mumtaz, ${ }^{2} \mathrm{~N}$ Hilmi, ${ }^{1} \mathrm{~L}$ Abu-Raddad. ${ }^{1}$ Weill Cornell Medical College in Qatar, Doha, Oatar; ${ }^{2}$ World Bank, USA

Background The Middle East and North Africa (MENA) continues to be perceived as a region with limited HIV epidemiological data. The objective of this work was to review and synthesise for the first time all available data related to HIV in Lebanon, in order to assess the status of the epidemic in this country and provide the basis for future interventions, prevention, and research needs. 